Schadstoffe in der Luft werden mit der Entstehung chronischer Erkrankungen, wie beispielsweise Herz-Kreislauf-Erkrankungen, in Verbindung gebracht. Für Diabetes allerdings liegen hierzu bislang keine gesicherten Daten vor. Das Ziel der Studie von Elisabeth Thiering und Dr. Joachim Heinrich, vom Institut für Epidemiologie I am Helmholtz Zentrum München, war, mögliche Assoziationen von Luftverschmutzung und einer Resistenz gegenüber Insulin bei Kindern zu identifizieren. Dazu werteten die Wissenschaftler Daten und Blutproben von 397 10-jährigen Kindern innerhalb einer prospektiven Kohortenstudie in Deutschland aus. Für die Erhebung der Feinstaubbelastung wurden Analysen der Verkehrsemissionen und der Bevölkerungsdichte in Wohnortnähe herangezogen. Zudem wurden die Modelle für die Einbeziehung weitere Daten angepasst, darunter sozioökonomischer Status der Familie, Exposition gegenüber Passiv-Rauchen sowie Geburtsgewicht, Entwicklungsstatus und BMI (Body-MassIndex) der Kinder. Die Untersuchungen ergaben, dass eine höhere Insulinresistenz bei jenen Kindern vorlag, die vermehrt Feinstäuben ausgesetzt waren. Pro $10,6 \mu \mathrm{g} / \mathrm{m}^{3}$ zusätzlichem Luftgehalt an Stickstoffdioxid $\left(\mathrm{NO}_{2}\right)$ stieg die Häufigkeit der Insulinresistenz um 17\%. Für Feinstaub in der Luft (bis zu einem Durchmesser von $10 \mu \mathrm{m}$ ) kam es zu einem Anstieg der Insulinresistenz um $19 \%$ pro $6 \mu \mathrm{g} / \mathrm{m}^{3}$. Auch die Entfernung der Wohnung zu einer stark befahrenen Straße spielte eine Rolle: in ihrer Nähe stieg die Insulinresistenz um 7\% pro 500 Meter.

Oxidativer Stress als mögliche Ursache „Die Toxizität von Luftschadstoffen ist unterschiedlich, allerdings sind sie alle potenzielle Oxidanzien. Das heißt, sie können direkt Lipide oder Proteine oxidieren oder auch indirekt oxidierende Signalwege in den Zellen aktivieren“, erklärt Thiering, Erstautorin der Studie. „Dieser oxidative Stress durch Feinstäube kann eine Erklärung für die Entwicklung einer Insulinresistenz sein. Zudem haben frühere Studien gezeigt, dass eine Ver- mehrung von kleinsten Luftpartikeln sowie $\mathrm{NO}_{2}$ zu einer Erhöhung entzündlicher Biomarker führen, was ebenfalls ein möglicher Trigger für eine Insulinresistenz sein kann.“

Die Nachbeobachtung der Kohorten erfolgt über insgesamt 15 Jahre. Aktuell untersuchen die Wissenschaftler, inwiefern sich ihre Beobachtung bei den älteren Kindern, während und nach der Pubertät, widerspiegeln und ob beispielsweise ein Wohnortwechsel, aus dem sich eine Änderung der Feinstaubbelastung ergibt, Aufschluss bietet über die Bedeutung der frühkindlichen bzw. späteren Exposition. „Wir können momentan die klinische Relevanz des erhöhten Risikos für die Insulinresistenz durch Feinstaubbelastung nicht abschätzen. Die Resultate unterstützen aber die These, dass die Entwicklung eines Diabetes im Erwachsenenalter mit Umweltfaktoren früherer Lebensabschnitte zusammenhängt“, resümiert Heinrich, Letztautor der Studie.

Pressemitteilung Helmholtz Zentrum München vom 13.5.2013

\title{
Typ-2-Diabetes
}

\section{Früherkennung durch Untersuchung der Darmflora}

Während der gemeinsamen Entwicklung von Mensch und Mikroben wurde der menschliche Darmtrakt von einigen tausend Bakterienarten besiedelt, deren Gesamtgewicht ungefähr 1,5 Kilogramm ausmacht. „Die Gene der heutzutage im Darmtrakt gesunder Menschen lebenden Bakterien sind in der Summe zahlreicher als die des menschlichen Organismus“, so Prof. Michael Roden, Düsseldorf. Nach aktuellen Analysen umfasst das Erbgut des Bakterienbestands in der Darmflora rund 3,3 Millionen Gene, im Vergleich zu nur 23000 Genen des menschlichen Organismus.

Diabetiker haben veränderte Darmflora Untersuchungen haben gezeigt, dass die Darmflora einzelner Menschen in hohem Maß übereinstimmt. Auf der Grundlage des Genstammes der Darmbakterien können Einzelpersonen in 3 Gruppen, die sogenannten Enterotypen, eingeteilt werden, erklärt
Roden: „Jeder Enterotyp zeichnet sich durch ein anderes bakterielles Ökosystem aus, mit vorwiegendem Anteil von ,Bacteroides', ,Prevotella' oder ,Ruminococcus'. Personen können sich somit in der Zusammensetzung der Bakterienarten, den Enterotypen und den bakteriellen Genen unterscheiden." Solche Merkmale der menschlichen Darmflora seien von großem Interesse für die Erforschung von vorbeugenden und therapeutischen Ansätzen des Typ-2-Diabetes (T2D), erläutert Roden: „Anhand einer Studie, in der 345 Personen chinesischer Herkunft untersucht wurden, konnte gezeigt werden, dass Patienten mit Typ-2-Diabetes eine veränderte Darmflora aufweisen, in der bestimmte Bakterien mit ganz spezifischen Genen vermehrt vorkommen." Ein daraus abgeleiteter Mikrobiota-T2D-Index könnte zukünftig bei der Untersuchung bislang gesunder Menschen die Vorhersage einer Diabetes-Erkrankung mit rund 80\% Treffsicherheit ermöglichen.
Probiotika oder Stuhltransfer als mögliche Ansätze

Bei Menschen mit Adipositas und bereits bestehendem Typ-2-Diabetes scheint eine Wiederherstellung einer gesunden Darmflora bisher schwierig und meist nur vorübergehend erreichbar. „Allerdings führt die Verwendung von Probiotika im Tiermodell sowie in einer noch geringen Anzahl von klinischen Studien zu vielversprechenden Ergebnissen“, sagt Roden. Auch der sogenannte Fäkaltransfer könnte ein möglicher therapeutischer Ansatz sein, der bereits bei Clostridium difficile-Erkrankungen erfolgreich eingesetzt wird: Dabei werden Exkremente eines gesunden Menschen in den Darm des Erkrankten verpflanzt. „In einer Untersuchung führte die Übertragung von Darmbakterien schlanker Personen in den Darm von Personen mit metabolischem Syndrom bei diesen nach 6 Wochen zu einer Verbesserung der Insulinempfindlichkeit“, so Roden.

Pressemitteilung Pressestelle Diabetes Kongress 2013 